\title{
Formation, Stability, and Mobility of One-Dimensional Lipid Bilayers on Polysilicon Nanowires
}

Shih-Chieh J. Huang, Alexander B. Artyukhin ${ }^{1}$, Julio A. Martinez ${ }^{1,3}$, Donald J. Sirbuly, Yinmin Wang ${ }^{1}$, Jiann-Wen $\mathrm{Ju}^{2}$, Pieter Stroeve ${ }^{3}$, and Aleksandr Noy ${ }^{*}$

${ }^{1}$ Chemistry, Materials, and Life Sciences Directorate, Lawrence Livermore National Laboratory, Livermore, CA 94550, ${ }^{2}$ Department of Civil and Environmental Engineering, University of California Los Angeles, Los Angeles, CA 90095, ${ }^{3}$ Department of Chemical Engineering and Materials Science, University of California Davis, Davis, CA 95616

"Corresponding author, noyı@llnl.gov

\section{Supporting Information}

\section{Materials AND Methods}

Nanotube synthesis. To fabricate substrates for growing suspended single-walled carbon nanotubes (SWNTs) we first etch a $5-\mu \mathrm{m}$ wide, $10-\mu \mathrm{m}$ deep channel in a silicon chip using STS reactive ion etching and subsequently grow $100 \mathrm{~nm}$ to $1 \mu \mathrm{m}$ of wet thermal oxide on silicon to prevent interaction of iron catalyst with silicon. ${ }^{1}$ We deposit the iron catalyst ${ }^{2}$ for SWNT growth by dipping the substrates into 70 $\mathrm{mg} / \mathrm{l}$ solution of $\mathrm{Fe}(\mathrm{NO})_{3} \cdot 9 \mathrm{H} 2 \mathrm{O}$ in propanol-2 with subsequent rinse in propanol and hexane. Nanotubes were grown according to the previously published procedure. ${ }^{3}$ Briefly, we insert the samples in a 1 -in quartz tube inside a 1 -in Lindberg/Blue tube furnace and ramp the temperature up to $850^{\circ} \mathrm{C}$ at a rate of 
$40^{\circ} \mathrm{C} / \mathrm{min}$. During the heating period, the system maintained the gas flows of 600 sccm of argon and $400 \mathrm{sccm}$ of hydrogen. After allowing the system to stabilize for 5 minutes after it reached the targeted temperature of $850^{\circ} \mathrm{C}$, we added $5 \mathrm{sccm}$ of ethylene to the gas mixture for 10 minutes. After 10 min growth period the system cooled down in argon flow.

Silicon deposition. Before depositing silicon on suspended carbon nanotubes we coat them with a thin layer $(5 \mathrm{~nm})$ of titanium in the electron beam deposition system to promote adhesion ${ }^{4}$ and ensure uniform Si nucleation. We subsequently coat these samples with a layer of amorphous silicon ${ }^{5}$ in a low pressure chemical vapor deposition (LPCVD) system using $100 \mathrm{sccm}$ of silane flow through 5 -in quartz tube at 150 mTorr and $535^{\circ} \mathrm{C}$. This growth process produces uniform silicon films with the film thickness controlled by the deposition time (Figure $\mathrm{S} 1$ ).

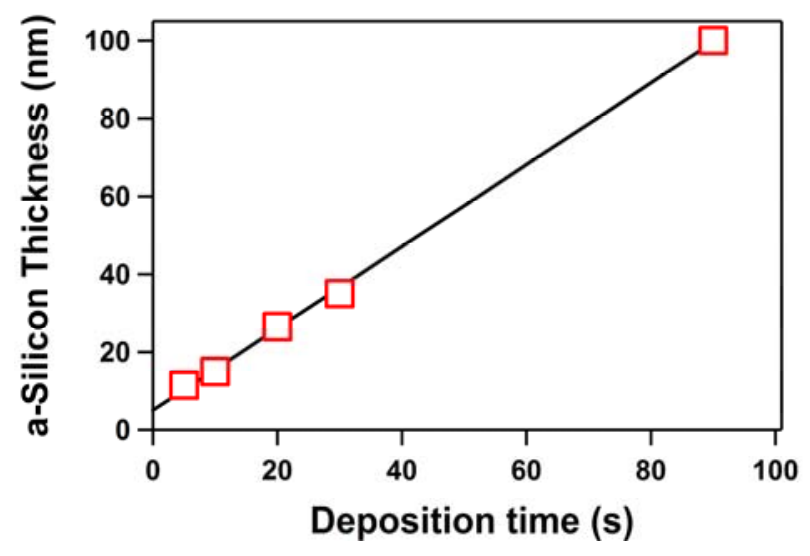

Figure S1. Calibration plot for the thickness of the amorphous $\mathrm{Si}$ coating deposited on the carbon nanotube samples. Black line represents a linear fit to the data $(R=0.9996)$. 
Lipid bilayer formation. We follow the previously described protocol $^{6}$ to prepare lipid vesicles from a mixture of DOPC, DOPE, and NBD-PE (Avanti Polar Lipids). We studied 5 lipid compositions: pure DOPC, 82\% DOPC + 18\% DOPE, 80\% $\mathrm{DOPC}+20 \%$ DOPE, $78 \%$ DOPC + 22\% DOPE, and 70\% DOPC + 30\% DOPE. We add 2 mol.\% of the fluorescent probe NBD-PE to the lipid mixture in all experiments. All vesicle preparations are in DI water. To form lipid bilayers on silicon nanowire substrates we expose them to the lipid vesicle solution for $30 \mathrm{~min}$. The samples are then thoroughly rinsed with copious amounts of water to remove loosely bound vesicles.

SEM and TEM imaging. SEM images were obtained using LEO Model 1560 scanning electron microscope. TEM images were recorded on a Philips $\mathrm{CM}_{3}$ ooFEG TEM operated at $300 \mathrm{kV}$ and equipped with a Gatan image filter system. The extraction voltage for the field-emission gun was $4.2 \mathrm{keV}$. The spatial resolution of the microscope is approximately 1.8 $\AA$. Samples for TEM were prepared on $\mathrm{Si}_{3} \mathrm{~N}_{4}$ grids from SPI Supplies.

Fluorescence imaging and FRAP measurements. To image lipid coating on silicon nanowires and measure lipid mobility we transferred the samples to a Nikon Eclipse TE3oo inverted confocal microscope equipped with a piezoelectrical translation stage and a Nikon Plan Apo 1ooX oil-immersion objective. ${ }^{7}$ We took care not to expose the samples to air after lipid deposition. To improve the time resolution for FRAP experiments we modified the setup described in the previous publication $^{8}$ to employ an Electro-Optical Modulator (Conoptics) to change the $\mathrm{Ar}$ 
laser beam power $(488 \mathrm{~nm})$ between bleaching $(100 \mu \mathrm{W})$ and imaging $(1 \mu \mathrm{W})$ modes. One FRAP cycle consists of bleaching the center of a lipid-coated nanowire for $8 \mathrm{~s}$ and monitoring subsequent fluorescence recovery for $8 \mathrm{~s}$. We typically perform 5 cycles of bleaching and recovery for each nanowire to obtain a diffusion coefficient. Each reported diffusion coefficient represents an average of data obtained on at least five individual nanowires.
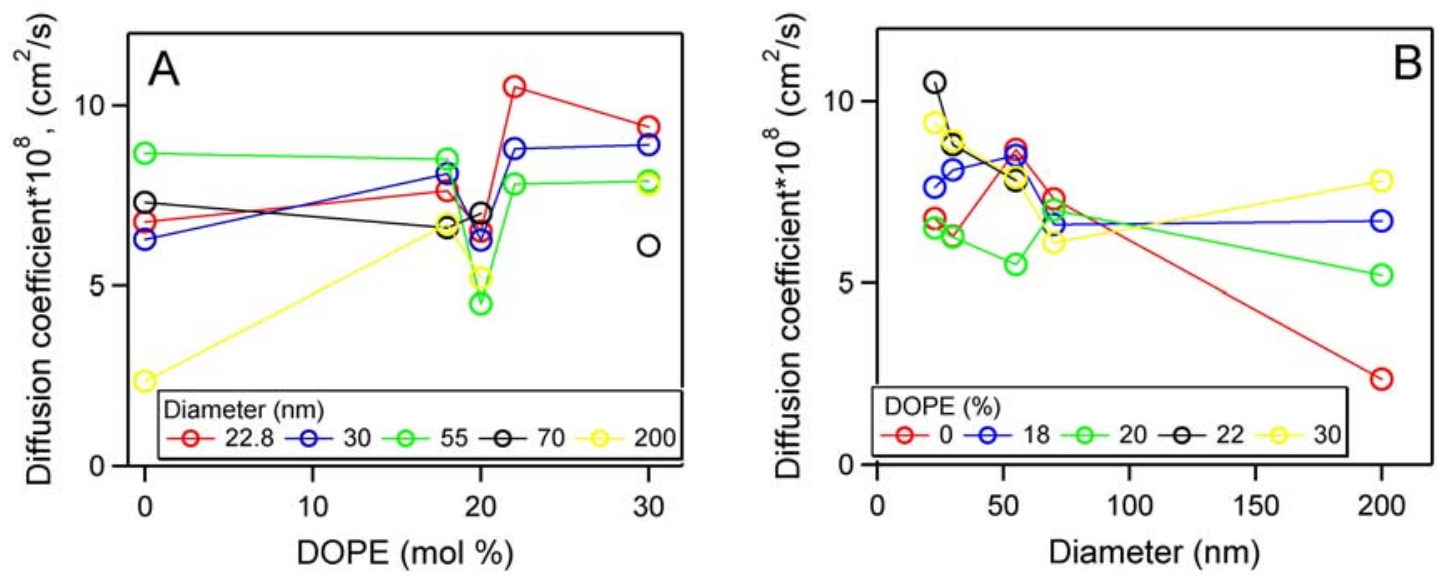

Figure S2. Diffusion coefficients of mixed DOPC-DOPE lipid bilayers supported on silicon nanowires as a function of (A) DOPE concentration or (B) nanowire diameter.
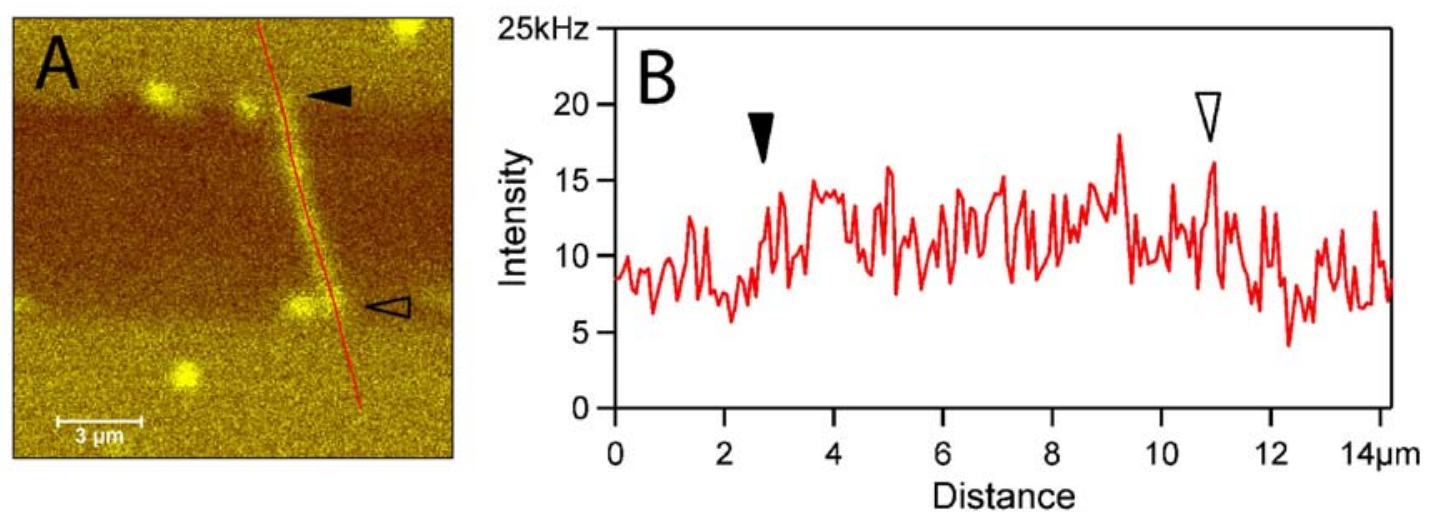

Figure S3. Line profile of the fluorescence image of a lipid coated nanowire stretched across the channel. (A) Scanning confocal microscopy image (same as in 
Figure $2 \mathrm{~B}$ in the main text). (B) Intensity profile along the red line in (A). Solid and open black triangle marks indicate the start and end of the nanowire.

\section{THERMODYNAMICS OF BILAYER FORMATION.}

To rationalize our results on formation of mixed DOPC-DOPE lipid membranes on nanowire substrates we consider thermodynamics of the bilayer formation on a curved surface. The free energy of formation of a one-component bilayer on a curved silicon nanowire surface (relative to the free energy of a lipid vesicle, $\Delta G^{V}$ ) $\Delta G$ includes two terms: (1) the bending energy change $\Delta E_{\text {bend }}$ associated with membrane shape transition from a spherical vesicle to a cylindrical nanowire and (2) the adhesion energy of the lipid headgroups to the silicon oxide surface of the nanowire, $E_{a d h}:^{9}$

$$
\Delta G=\Delta G^{N W}-\Delta G^{V}=\Delta E_{\text {bend }}-E_{\text {adh }}=E_{\text {bend }}^{N W}-E_{\text {bend }}^{V}-E_{\text {adh }} .
$$

Neglecting the Gaussian curvature term, the bending energy per unit area of the lipid membrane is equal to ${ }^{10-12}$

$$
E_{\text {bend }}=\frac{k}{2} \cdot\left(\frac{1}{R_{1}}+\frac{1}{R_{2}}-J_{0}\right)^{2}
$$

where $k$ and $J_{o}$ are the bending modulus and the intrinsic curvature of the lipid monolayer, respectively, and $R_{1}$ and $R_{2}$ are the two principal radii of curvature of the membrane. ${ }^{13}$ When considering bending of a two-component membrane, we also need to take into account an entropic contribution to the free energy:

$$
\Delta G=\Delta E_{\text {bend }}-T \Delta S-E_{\text {adh }}=E_{\text {bend }}^{N W}-E_{\text {bend }}^{V}-T \cdot\left(\Delta S_{\text {mix }}^{N W}-\Delta S_{\text {mix }}^{V}\right)-E_{\text {adh }} .
$$


In a flat symmetrical bilayer both leaflets are identical and lipid components are homogeneously distributed. Membrane bending breaks the symmetry with the two leaflets now having curvatures of the opposite signs. Conical molecules (such as DOPE) with higher intrinsic curvature prefer to reside in the inner leaflet. ${ }^{14,}{ }^{15}$ Thus lipid rearrangement between the two leaflets would be favored energetically, but would also incur a penalty due to the decrease of the mixing entropy. It is this balance between bending energy and mixing entropy that ultimately determines degree of lipid redistribution upon bending. ${ }^{\dagger}$ To determine equilibrium degree of redistribution (Figure $S_{4}$ a) we minimize the free energy $\Delta G^{N W}=E_{b e n d}^{N W}-T \Delta S_{\text {mix }}^{N W}$ with respect to the DOPE fraction in the inner monolayer $\left(x_{D O P E}^{\text {inner }}\right)$ for each nanowire radius $R$ and each nominal DOPE concentration $x_{o}$ in the DOPC-DOPE mixture

$$
\left.\frac{\partial \Delta G^{N W}}{\partial x_{D O P E}^{\text {inner }}}\right|_{R, x_{0}}=0
$$

We repeat the same procedure for lipid redistribution in a spherical vesicle and then use these optimized redistribution values for a cylindrical bilayer and a spherical vesicle to calculate $\Delta G$ using Equations 1, 3. Our calculation used the following parameters: $k=3 \cdot 9 \cdot 10^{-20} \mathrm{~J},{ }^{16-20} E_{a d h}=1.5 \cdot 10^{-4} \mathrm{~J} / \mathrm{m}^{2},{ }^{21-23} \quad J_{0}^{\text {DOPC }}=-0.05 \mathrm{~nm}^{-1}$, $J_{0}^{\text {DOPE }}=-0.33 \mathrm{~nm}^{-1.13,}{ }^{24}$ For each DOPC-DOPE mixture we calculated the effective intrinsic curvature of both inner and outer leaflets using a linear combination ${ }^{11}$ of intrinsic curvatures for pure DOPC and DOPE monolayers. The DOPE headgroup

\footnotetext{
${ }^{\dagger}$ In the intact bilayer lipid distribution between leaflets maybe kinetically frozen due to slow flip-flopping process. Thermodynamic control should prevail during vesicle fusion when transient formation of open edges makes fast flip-flop possible.
} 
is less hydrated than DOPC, ${ }^{25}, 26$ and should produce stronger adhesion to the substrate, but in lipid mixtures we expect this effect to be noticeable only for DOPE concentrations higher than ca. 60 mol.\%. ${ }^{27}$ Therefore for all lipid compositions studied in this work we used the adhesion energy of pure DOPC. We also assumed that adhesion energy is independent of the substrate curvature.
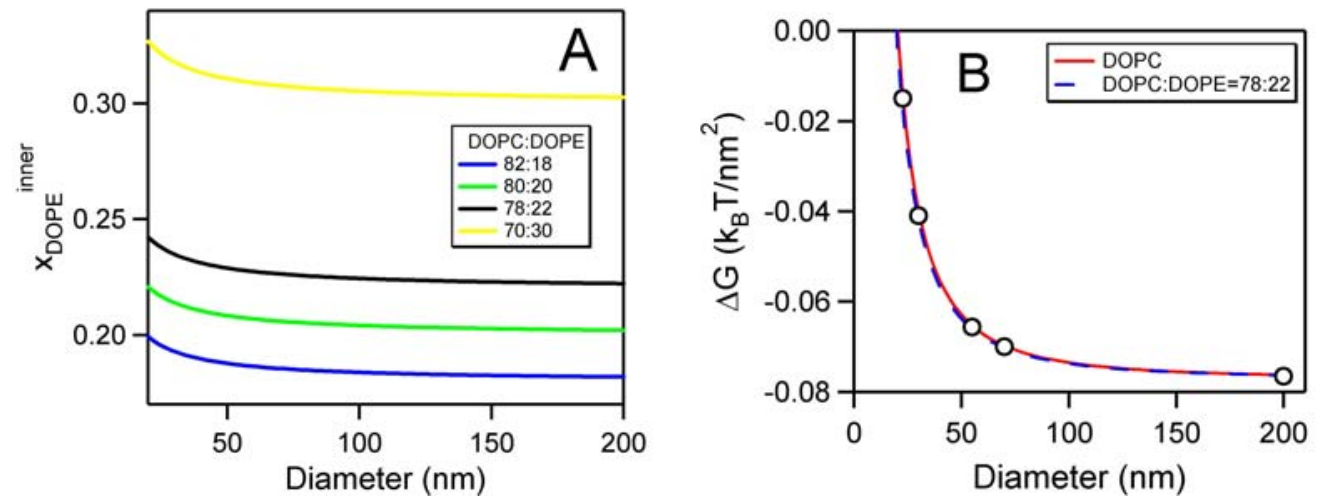

Figure S4. (A) Enrichment of DOPE in the inner monolayer of nanowiresupported lipid bilayer. (B) Free energy of formation of a nanowire-supported lipid bilayer from 6o-nm vesicles as a function of nanowire radius for two lipid compositions. Circles correspond to the sizes of the nanowires used in this work.

Remarkably, even if we take into account equilibrium lipid redistribution, the calculated bilayer formation free energies do not show much variation for any of the lipid compositions (calculated $\Delta G$ profiles essentially coincide, Figure $\mathrm{S}_{4} \mathrm{~b}$ ). This behavior implies that from a thermodynamics point of view both a mixed DOPC:DOPE (70:30) bilayer and a pure DOPC are stable, which is consistent with our experimental observations. We also note that free energy of formation of nanowire-supported bilayer strongly depends on the size of the parent vesicles: the smaller the vesicles the more unstable they are due to high bending energy and the 
more negative $\Delta G$ becomes. Our calculations show that formation of a supported membrane on nanowires with diameters down to $20 \mathrm{~nm}$ is energetically favorable if the vesicle diameter is less than $60 \mathrm{~nm}$ (Figure $\mathrm{S}_{4} \mathrm{~b}$ ). Note that the free energy curves do not have a minimum indicating that the flat bilayer represents the lowest energy configuration for all studied membranes. Our conclusion is that the addition of cone-shaped DOPE molecules does not lead to a significant stabilization of highly curved membranes: energy gained due to lipid rearrangement according to their intrinsic curvatures cannot overcome the entropic penalty imposed by segregation of the lipid molecules. ${ }^{28}$

\section{MOBILITY OF ONE-DIMENSIONAL LIPID BILAYERS ON CRYSTALLINE VLS SILICON NANOWIRES.}

Nanowire synthesis and substrate preparation. p-type silicon nanowires (SiNWs) were produced by chemical vapor deposition following a previously described method. ${ }^{29}$ Briefly, 30-nm colloidal gold nanoparticles (Ted-Pella) were deposited onto a silicon substrate that was previously functionalized with poly Llysine, (o.1\% aqueous solution, Ted-Pella) to provide an overall positively charged surface for colloidal gold adsorption. After deposition the silicon substrate was cleaned with oxygen plasma to remove organic materials. SiNW synthesis was carried in a 1-in tube furnace at $460^{\circ} \mathrm{C}$ using a mixture of $\mathrm{SiH}_{4}(10 \%$ in $\mathrm{He}, 30 \mathrm{sccm})$ and $\mathrm{B}_{2} \mathrm{H}_{6}$ (100 ppm in He, $\left.2 \mathrm{sccm}\right)$ under a total pressure of 100 Torr for 20 minutes. Once the synthesis was completed, the resulting crystalline SiNWs (Figure $\mathrm{S}_{5} \mathrm{~A}$ ) were transferred into solution phase by sonication of the silicon 
substrate for 10 second in ethanol. TEM characterization showed that the diameter of the nanowires produced by this method varied between 20 and $40 \mathrm{~nm}$. We did not perform any further fractionation of the nanowires by size for the subsequent experiments. To obtain nanowires suspended across the openings of the TEM grid approximately ten $1-\mu \mathrm{L}$ drops of this solution were deposited onto a copper TEM grid (200o mesh, Ted-Pella). To obtain lipid bilayers on the silicon nanowires the TEM grids were immersed in the aqueous suspension of lipid vesicles according to the aforementioned procedure for the formation of supported lipid bilayers on nanowire substrates.

Formation and mobility of lipid bilayers on VLS SiNWs. The same vesicle fusion technique used for producing one-dimensional lipid bilayers on amorphous silicon nanowires readily produces them on crystalline VLS-grown silicon nanowires (VLS-SiNWs) (Figure $\mathrm{S}_{5} \mathrm{~B}$ ). This result is not surprising, since both types of nanowires have similar curvatures and similar silicon oxide surface termination. To characterize the lipid mobility on VLS-SiNWs we have performed FRAP experiments using protocols identical to what we used to measure lipid mobility on amorphous SiNWs. One-dimensional lipid bilayers on VLS-SiNWs show very good recovery levels (Figure $\mathrm{S}_{5} \mathrm{C}$ ) which are similar to what we observed on the amorphous SiNWs. Significantly, the measured diffusion coefficients $\left(\mathrm{D}=(8.4 \pm 0.3) \cdot 10^{-8} \mathrm{~cm}^{2} / \mathrm{s}\right)$ are in good agreement with the diffusion coefficients measured for the amorphous SiNWs in the size range of the VLS-SiNWs used for this study. Thus, we conclude that minor differences in surface roughness 
originating in the different synthetic procedures used in this study do not produce any significant differences in the mobility of one-dimensional lipid bilayers adsorbed on these substrates.
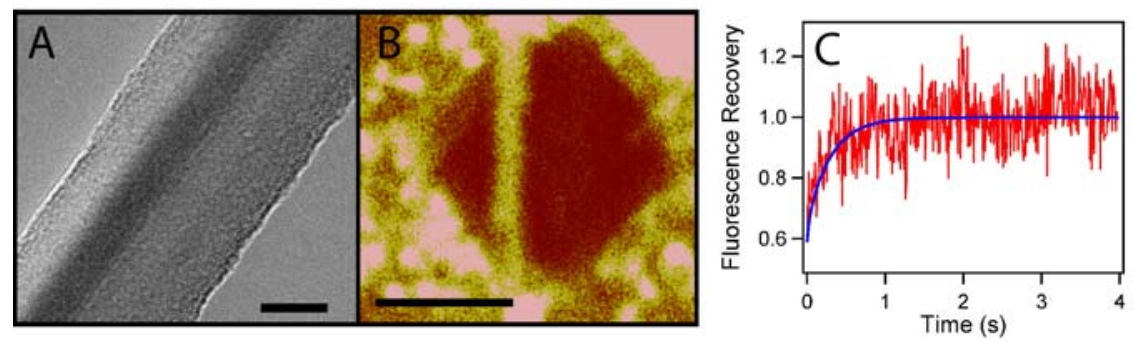

Figure S5. (A) TEM image of a VLS-grown SiNW. Scale bar: $10 \mathrm{~nm}$. (B) Scanning confocal fluorscence micrograph of a one-dimensional lipid bilayer formed on a VLS SiNW stretched across a hole in the TEM grid. Scale bar: $5 \mu \mathrm{m}$. (C) An experimental fluorescence recovery curve (red) recorded for a FRAP measurement on the lipid-coated VLS SiNW. The bleaching conditions were the same as used for amorphous SiNW experiments. Blue curve indicates the fit to the diffusion model with the diffusion coefficient of $8.5 \cdot 10^{-8} \mathrm{~cm}^{2} / \mathrm{s}$.

\section{Acknowledgements.}

We thank Gengfeng Zheng (Lieber Group, Harvard University) for teaching us the basic procedures for the VLS growth of silicon nanowires.

\section{References.}

1. Simmons, J. M.; Nichols, B. M.; Marcus, M. S.; Castellini, O. M.; Hamers, R. J.; Eriksson, M. A. Small 2006, 2, 902-909.

2. Hafner, J. H.; Cheung, C. L.; Oosterkamp, T. H.; Lieber, C. M. J. Phys. Chem. B 2001, 105, 743-746.

3. Artyukhin, A. B.; Bakajin, O.; Stroeve, P.; Noy, A. Langmuir 2004, 20, 1442-1448.

4. Zhang, Y.; Franklin, N. W.; Chen, R. J .; Dai, H. J . Chem. Phys. Lett. 2000, 331, 35-41. 
5. Ibok, E.; Garg, S. J . Electrochem. Soc. 1993, 140, 2927-2937.

6. Zhang, L. Q.; Longo, M. L.; Stroeve, P. Langmuir 2000, 16, 5093-5099.

7. Noy, A.; Huser, T. R. Rev. Sci. Instr. 2003, 74, 1217-1221.

8. Artyukhin, A. B.; Shestakov, A.; Harper, J .; Bakajin, O.; Stroeve, P.; Noy, A. J. Amer. Chem. Soc. 2005, 127, 7538-7542.

9. Ponnuswamy, A.; Nulton, J .; Mahaffy, J . M.; Salamon, P.; Frey, T. G.; Baljon, A. R. C. Phys. Biol. 2005, 2, 73-79.

10. Helfrich, W. Z. Naturforsch. 1973, 28c, 693-703.

11. Hamai, C.; Yang, T.; Kataoka, S.; Cremer, P. S.; Musser, S. M. Biophys. J. 2006, 90, 1241-1248.

12. Parthasarathy, R.; Groves, J. T. Soft Matter 2007, 3, 24 - 33.

13. Zimmerberg, J .; Kozlov, M. M. Nat. Rev. Mol. Cell Biol. 2006, 7, 9-19.

14. Litman, B. J. Biochemistry 1973, 12, 2545-2554.

15. Litman, B. J . Biochemistry 1974, 13, 2844-2848.

16. Rawicz, W.; Olbrich, K. C.; McIntosh, T.; Needham, D.; Evans, E. Biophys. J. 2000, 79, 328-339.

17. Booth, P. J .; Templer, R. H.; Meijberg, W.; Allen, S. J .; Curran, A. R.; Lorch, M. Crit. Rev. Biochem. Mol. Biol. 2001, 36, 501-603.

18. Evans, E.; Needham, D. J . Phys. Chem. 1987, 91, 4219-4228.

19. Attard, G. S.; Templer, R. H.; Smith, W. S.; Hunt, A. N.; J ackowski, S. Proc. Natl. Acad. Sci. U.S.A. 2000, 97, 9032-9036.

20. Chen, Z; Rand, R. P. Biophys. J. 1997, 73, 267-276.

21. Furukawa, K.; Sumitomo, K.; Nakashima, H.; Kashimura, Y.; Torimitsu, K. Langmuir 2006, 23, 367-371.

22. Nissen, J .; Gritsch, S.; Wiegand, G.; Radler, J . O. Eur. Phys. J . B 1999, 10, 335-344.

23. Schonherr, H.; J ohnson, J . M.; Lenz, P.; Frank, C. W.; Boxer, S. G. Langmuir 2004, 20, 11600-11606.

24. Marsh, D. Biophys. J . 1996, 70, 2248-2255.

25. Marra, J .; Israelachvili, J . Biochemistry 1985, 24, 4608-4618.

26. McIntosh, T. J . Chem. Phys. Lipids 1996, 81, 117-131.

27. Evans, E.; Needham, D. J. Phys. Chem. 1987, 91, 4219-4228. 
28. Cooke, I. R.; Deserno, M. Biophys. J . 2006, 91, 487-495.

29. Patolsky, F.; Zheng, G.; Lieber, C. M. Nat. Protocols 2006, 1, 1711-1724. 